This is a pre-copyedited version of a contribution published in Research Proceedings 2018 (Eds: Bernard Fortz, Martine Labbé), published by Springer Nature Switzerland AG.

\title{
User-based Redistribution in Free-floating Bike Sharing Systems
}

\author{
Christoph Heitz ${ }^{1}$, Roman Etschmann ${ }^{1}$, Raoul Stoeckle ${ }^{2}$, Thomas Bachmann ${ }^{3}$ and Mat- \\ thias Templ ${ }^{1}$ \\ ${ }^{1}$ Zurich University of Applied Sciences, School of Engineering, Winterthur, Switzerland \\ ${ }^{2}$ smide KmG, Uster, Switzerland, ${ }^{3}$ La Mobilière, Bern, Switzerland
}

\begin{abstract}
We investigate the problem of user-based redistribution for freefloating bike sharing systems (BSS). We present a stochastic model of the bike dynamics and we show that the spatial distribution of bikes is correlated. This is specific to free-floating systems and it results in a substantially reduced service level.

Offering incentives to users may stimulate them to change their behavior and usage pattern. We analyze drop-off incentives, derive an incentive methodology and study its potential. We show that by implementing a smart incentive system, the number of bikes for establishing a specific service level can be reduced significantly, even if only a minority of users participates. Under realistic behavioral assumptions, $30-50 \%$ reduction of bikes is achievable, which converts into substantial costs savings for the operator.

Our research was carried out in the context of the development of the new ebike sharing system "smide" in Zurich, launched in 2017. The incentive approach has been implemented and tested in a field test.
\end{abstract}

Keywords: bike sharing system, free-floating, redistribution, simulation.

\section{$1 \quad$ Introduction}

Free-floating bike sharing systems (BSS) are relatively new. In such systems, bikes can be dropped off at any location within a specified area. Bikes can be located and unlocked via smartphone. In recent years, free-floating BSS have been rapidly introduced worldwide, for example by oBike (China) or LimeBike (USA), and it seems as if the future of BSS are free-floating systems. In Zurich, the first free-floating e-bike system has been launched in 2017 by the insurance company La Mobilière and is now being operated by the company smide (www.smide.ch).

Free-floating systems avoid expensive docking stations and offer more flexibility. On the other hand, the redistribution of bikes from areas where they are dropped off to areas where they are needed is much more costly than for station-based systems, because the bikes are distributed over the complete area rather than concentrated at few stations. This is a particular critical issue since redistribution is typically the largest cost factor of a BSS even for station-based BSS (see, e.g. [1]). In order to reduce 
the redistribution costs, several authors have suggested to transfer at least a part of the redistribution task to the users, which is called user-based redistribution. Nearly all of the literature, however, is focused on station-based systems [e.g. 2-6], and to our knowledge nothing has been published on user-based redistribution for free-floating systems.

In this paper, we study the problem of user-based redistribution for free-floating BSS. We study the dynamics of flee-floating systems, and we assess the potential of dynamical user incentivation for generating user-based redistribution. The presented results have been derived during the development of the smide system, where userbased redistribution is a core element. Smide has been developed in a cooperation of the Zurich University of Applied Sciences and La Mobilière. The incentive approach has been tested in a field test in fall 2017, and is implemented in the current smide system.

\section{Free-floating BSS: Dynamics and user incentives}

\subsection{Basic dynamical model of free-floating BSS}

For analyzing user incentivation in BSS, we study a quadratic area A of $3 \mathrm{~km} \times 3 \mathrm{~km}$, discretized in quadratic cells of width $\mathrm{c}$. We chose $\mathrm{c}=100 \mathrm{~m}$, but a finer grid can be used. Locations are specified by indices $(\mathrm{i}, \mathrm{j})$. The number of bikes is denoted by $\mathrm{N}$. Demand for bikes generally depends on both time and location. For this paper, we restrict ourselves to constant and homogenous demand, thus in each cell we have the same demand rate.

If a demand occurs in cell (i,j), the user is willing to walk a certain distance to pick up an available bike. Empirical results show that the willingness to walk for picking up a bike is about $300-500 \mathrm{~m} \mathrm{[4].} \mathrm{We} \mathrm{use} \mathrm{a} \mathrm{simple} \mathrm{behavioral} \mathrm{model} \mathrm{assuming} \mathrm{that} \mathrm{all}$ users are willing to walk a maximum distance of 2 cells in each direction. Thus, a user in cell $(\mathrm{i}, \mathrm{j})$ is willing to pick up a bike within the region $\mathrm{U}(\mathrm{i}, \mathrm{j})$ consisting of all cells $\left(i^{\prime}, j^{\prime}\right)$ with $i^{\prime} \in[i-2, i+2]$, and $j^{\prime} \in[j-2, j+2]$. The user picks the next available bike within $U$. A service violation occurs if $U$ is empty.After the ride, the bike is dropped off in a random cell.

The state of the system is given by the bike distribution $n(i, j, t)$. Its is given by the following process: Demand occurs as a Poisson process in a random cell. If a bike in the environment is available, it is removed. The destination cell is chosen randomly and the bike is dropped off immediately. Incentives might change the drop-off location. Similar to [4] and [5], we define the service level $\beta$ as the probability that a demand is met, i.e. that a bike is available within the walking distance.

The studied dynamical system is balanced: pick-up rates and drop-off rates are equal in each cell. This is unrealistic, of course, but we chose this configuration because it allows to study the effect of user-based redistribution directly, without the need of explicitly modeling the operator's redistribution activity. The modeled situation mimics a redistribution strategy where the operator focuses his redistribution 
activity on counterbalancing macroscopic and systematic flows, but ignores the stochastic fluctuations of the bike distribution.

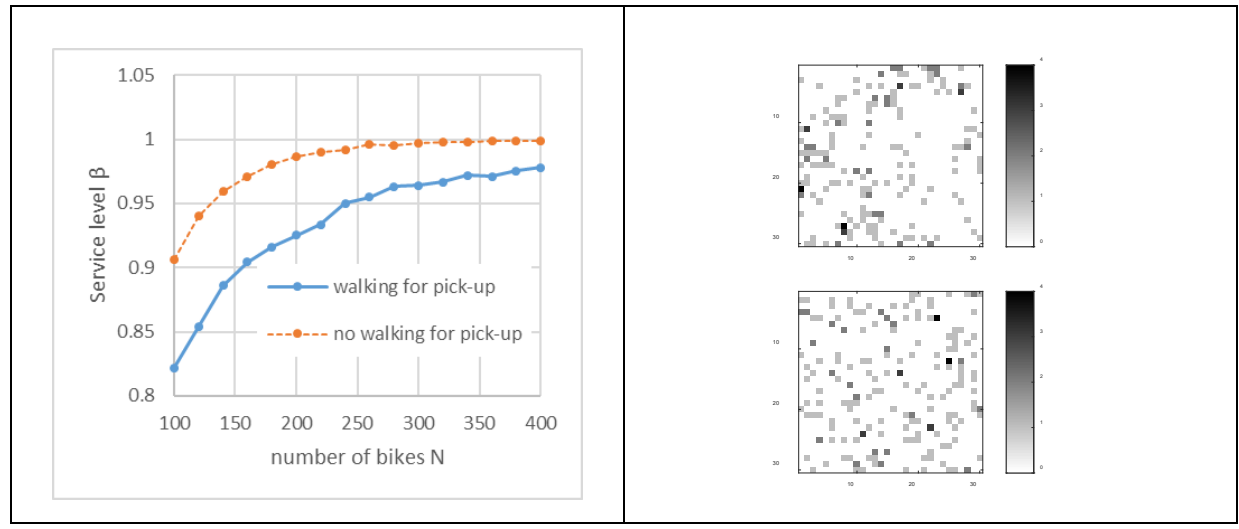

Fig. 1. Left: Service level as a function of number of bikes $\mathrm{N}$ with users walking to pick up a bike (blue solid line) vs. not walking (orange dotted line). Right: Sample bike distributions for $\mathrm{N}=200$ for walking users (top) vs. non-walking users (bottom).

\subsection{Service level and system dynamics of free-floating BSS without incentives}

As a reference case, we study a system without any user incentives by simulating the above specified process with 50000 demand events. The trip time has been set to zero for simplicity. In Fig. 1 (left), the service level is shown as a function of the number of bikes $\mathrm{N}$. For $\mathrm{N}=100$ bikes $\left(11.1\right.$ bikes $\left./ \mathrm{km}^{2}\right)$, the service level is $82 \%$, and it approaches $97 \%$ for $\mathrm{N}=400$.

Note that the spatial distribution of bikes shows a strong spatial correlation of neighboring cells: The probability of a cell being empty is higher if the neighboring cells are empty, and vice versa. A result is the appearance of large starved areas (Fig. 1 right, top). This is caused by the following mechanism: Users within a starved area walk to the border of the area to pick up a bike. Thus, the demand for bikes just outside of a starved area is higher than on average, and a starved area tends to grow. In addition, if a bike is being dropped in the middle of a starved area, it collects all the demand of the neighboring cells, and is being picked up much faster than on average, making starved areas more persistent. Thus, the fact that users walk to pick up a bike creates a bike distribution that shows large empty areas combined with overpopulated areas elsewhere. In Fig. 1, right, bottom, the bike distribution is shown for the case where users would not be willing to walk. It can be seen that the distribution is much more regular, and there are much fewer areas where no bike can be found within U. The corresponding service level is much higher, (Fig. 1, left, orange dotted line). Thus, the pick-up walking of users has a significant impact on the service level by creating this spatial correlation. It is specific to free-floating BSS, and is not present in station-based BSS. 


\subsection{Incentive system: value function}

User incentives for improving the bike distribution may be offered in different ways: Users may be motivated to drop off bikes in starved areas, which leads to an immediate service level improvement, or to pick up bikes in overpopulated areas, hoping that the bike increases the service level at the drop-off location, or a combination of both (giving incentives for a specific trip). In this paper and as in [5], we only study dropoff incentives, as they are the most direct way for improving service level.

The goal of such an incentive system is an improvement of the service level by influencing where users drop off their bikes after usage. A dropped bike at location $(\mathrm{i}, \mathrm{j})$ has a positive impact on the service level if and only if it avoids a service failure in the future. The earlier the bike is needed, the more value is generated by the drop-off in terms of service level. Thus, we base our incentive approach on the time interval $\mathrm{Z}(\mathrm{i}, \mathrm{j})$ until a bike dropped at $(\mathrm{i}, \mathrm{j})$ will actually be needed, i.e. the time until a service failure would occur if the bike was not dropped.

$\mathrm{Z}(\mathrm{i}, \mathrm{j})$ is a random variable and depends on the current bike distribution as well as the future demand and the dropping behavior. We define a value function $v(i, j)$ as the reciprocal of the expectation value of $\mathrm{Z}(\mathrm{i}, \mathrm{j}): v(i, j)=(E(Z(i, j)))^{-1}$. So, v(i,j) is a measure that directly reflects the impact of a drop at $(\mathrm{i}, \mathrm{j})$ on the service level. Maximizing this value for each ride would lead to a minimization of service failures and, equivalently, to an improvement of the service level.

The quantity $E(Z(i, j))$ is well defined. It could be determined by forwardsimulating the system starting from the current bike distribution until the next service failure occurs due to a demand event at (i',j'), where (i',j') $\in U(i, j)$. By averaging many simulation runs, the expectation value $E(Z(i, j))$ could be determined. This, however, is numerically cumbersome. In the following we derive a method of calculating an approximation of $E(Z(i, j))$.

Let $B\left(i^{\prime}, j^{\prime}\right)$ the time to the next service failure created by a demand event in cell $\left(i^{\prime}, j^{\prime}\right) \in U(i, j)$. The time to the next service failure that could be avoided by dropping a bike at $(i, j)$ is the minimum of all these $B\left(i^{\prime}, j^{\prime}\right)$. If the $B\left(i^{\prime}, j^{\prime}\right)$ were exponentially distributed random variables, then the minimum of a set of such variables would be the exponentially distributed, where the rate is a the sum of the rates of the $B\left(i^{\prime}, j^{\prime}\right)$. This motivates setting the value $\mathrm{v}(\mathrm{i}, \mathrm{j})$ to

$$
v(i, j)=\sum_{\left(i^{\prime}, j^{\prime}\right) \in U(i, j)} \frac{1}{E\left(B\left(i^{\prime}, j^{\prime}\right)\right)}
$$

For calculating E(B(i',j')), we approximate the dynamics of the full system by restricting the dynamical analysis to the environment $U\left(i^{\prime}, j^{\prime}\right)$. Let $n(t)$ be the number of bikes in this area, with $\mathrm{n}(0)$ the current number of bikes, calculated by summing up the current bike distribution in $\mathrm{U}\left(\mathrm{i}^{\prime}, \mathrm{j}^{\prime}\right)$. We assume that $\mathrm{n}(\mathrm{t})$ is a stochastic birth-deathprocess with a birth rate $\lambda_{U}$ and death rate $\mu_{U}$, where $\lambda_{U}$ is the aggregation of the demand in $U\left(i, j^{\prime}\right)$, and $\mu_{U}=\beta \cdot \lambda_{U}$, where $\beta$ is the service level. The expectation value of $B\left(i, j^{\prime}\right)$ can then be calculated as a mean first passage time of an equivalent Markov model with absorbing state -1 . In general, the transient probabilities can be calculated 
by integrating the Komogorov forward equations. For the special case of constant rates, the expectation value can be calculated analytically [7]

$$
E\left(B\left(i^{\prime}, j^{\prime}\right)\right)=\frac{n(0)-1}{\lambda_{U}-\mu_{U}}=\frac{n(0)-1}{(1-\beta) \lambda_{U}} .
$$

Inserting this in Eq. (1), the value $v(i, j)$ of dropping off a bike at a specific location $(\mathrm{i}, \mathrm{j})$ can be calculated for each location.

\subsection{Incentive system: rewards for triggering behavioral changes}

The goal of each incentive system is to change the behavior of the users for improving the service level. In this paper, our aim is not to analyze a specific incentive system, but to assess the potential of an incentive system based on the value function $\mathrm{v}(\mathrm{i}, \mathrm{j})$ as defined in Section 2.3, in a most general way. We assume that the value map $\mathrm{v}(\mathrm{i}, \mathrm{j})$ is mapped into a reward function $\mathrm{r}(\mathrm{i}, \mathrm{j})$ where $\mathrm{r}$ is strictly monotonically increasing with $\mathrm{v}$, and the re ward function is communicated continuously to all users via their smart phone. This is in contrast to [4] and [5], where drop-off rewards are userspecific and offered only after the true destination is known, which leads to complex implementation issues. Our approach offers a reward for each trip in advance, and the reward only depends on the dropping location. This leads to additional expenses, as rewards are also given if the user does not change her behavior. On the other hand, such a system may trigger additional rides to starved areas. Is shown in section 2.5, this contributes substantially to the improvement of the service level.

There are still many degrees of freedom for concrete implementation, for example the type of the reward (e.g. monetary refund or free minutes for the next ride), its level, and how exactly the mapping between $\mathrm{v}$ and $\mathrm{r}$ is defined. For our analysis we only assume that users are offered more reward if $\mathrm{v}$ is higher, and that the reward is sufficiently high to influence the users' behavior. Based on empirical findings in the field test of smide, we assume that offering a location-based drop-off incentive has two different behavioral effects: (a) Users may drop off their bike somewhere close to, but not directly at, their destination, if they can increase their reward by doing so. So, they would accept to take a short walk at the end of the trip for increasing their reward. (b) The offered rewards might trigger additional trips. For example, users may choose to use the BSS instead of public transport, just because they can earn a reward which can be used for future BSS trips.

We assume furthermore that a user either ignores the offered reward altogether, or he changes his behavior such that his reward is maximized. For case (a), a user with destination (i,j) would drop the bike at the cell $\left(i^{\prime}, j^{\prime}\right) \in U(i, j)$ where $v$ is maximum. For case (b), the user would make a trip with a destination corresponding to the global maximum of $v$. Note that, in contrast to [4] and [5], we do not try to calculate explicitly the optimum level of the reward. The percentage of users responding to the incentive system is a model parameter which accounts for the dependence of behavioral change on the reward magnitude, averaged over all users. Optimizing the way of setting the height of the rewards with methods like in [4,5] might reduce the costs of the 
incentive system, but would not substantially change the overall effect of the incentive, which is what we are focusing on in this paper.

Using these behavioral assumptions, we are able to generate quantitative results about the potential of an incentive system based on the value function $\mathrm{v}$, without the need to specify exactly how the value map is transferred into corresponding rewards.

\subsection{Simulation results}

We simulate the user behavior under the above described incentive scheme. Trips are generated as described in section 2.1 . With a probability $\mathrm{p}_{1}$, the user is reacting to the incentive by dropping the bike in the environment $U(i, j)$ around his destination $(i, j)$, choosing the cell with the highest value. In addition, a proportion $\mathrm{p}_{2}$ of additional trips are generated. For example, $\mathrm{p}_{2}=0.1$ means that $10 \%$ additional trips are generated by offering the incentives, compared to not offering incentives at all. For these additional trips, we assume that the pick-up distribution is the same as before, but the drop-off location is the location with the highest reward within the whole area. This is motivated by our empirical findings with users of smide, indicating that additional trips are generated by high rewards only.

In Fig. 2, the service level for different parameter values are shown. It can be seen that both mechanism of behavioral change lead to substantial increase of the service level, and for achieving a given service goal, the number of needed bikes can be strongly reduced. For a service level goal of 0.95 , without user incentives, around 250 bikes would be needed for the simulated area of $9 \mathrm{~km}^{2}$. It can be seen that, even with a modest reaction of users to the incentives, a reduction of bikes by $50 \%$ can be obtained. For example, with $10 \%$ additional trips and a $30 \%$ user response for drop-off displacement of $300 \mathrm{~m}$, the number of bikes can be reduced by $50 \%$.

Of course this depends on the fraction of users that react to the incentives. Many parameters might influence this, such as level and type of incentive (e.g. free minutes vs. monetary reward), but also the service design and the user communication. In field tests of smide during fall 2017 in Zurich, we tested the response of the users to incentives consisting of $5 \ldots 15$ free minutes for future rides, offered at specified locations. This generated about $10 \%$ additional trips, so $\mathrm{p}_{2}=0.1$ seems realistic.

No empirical results are yet available for parameter $p_{1}$. In Fig. 2, right side, a sensitivity analysis with respect to $p_{1}$ is shown, indicating the number of bikes necessary to achieve a service level of $95 \%$ as a function of $p_{1}$, where $p_{2}$ is set to 0 . It can be seen that the effect of user participation is highly non-linear: Even a small part of the users can have a large impact on the system performance. In our case, a participation of $20 \%$ already yields $50 \%$ of the effect. This supports earlier findings (see, e.g. [4]).

The simulation results indicate that both behavioral effects as described in Section 2.3 seem to have a similar efficiency, but their mechanisms differ: Effect (a) is efficient because it reduces the spatial correlation. For this, even small changes of the dropping location are sufficient. Effect (b), on the other hand, leads to a large-scale redistribution of bikes. 

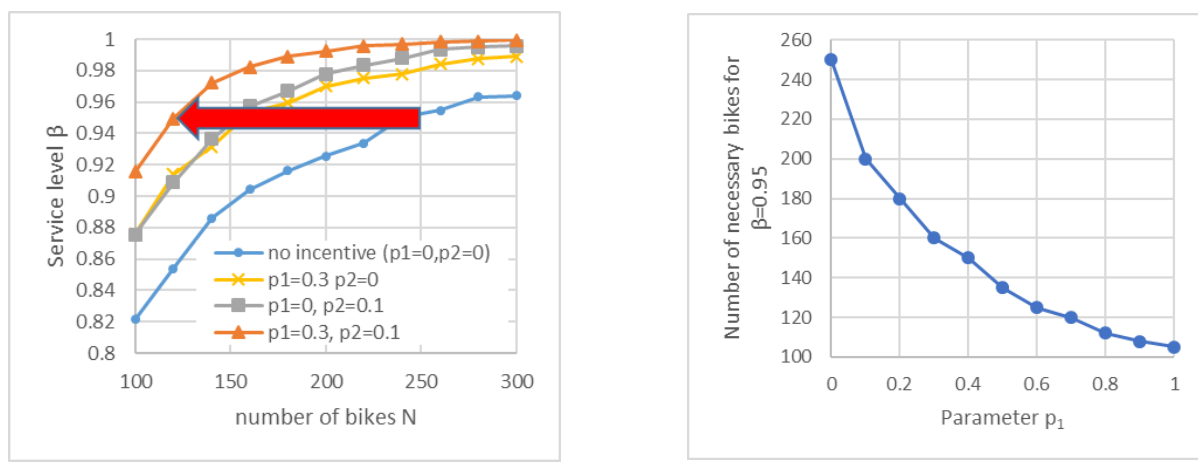

Fig. 2. Left: Service level as function of number of bikes for different parameter values of $p_{1}$ and $\mathrm{p}_{2}$. For a service level goal of $95 \%$, the number of necessary bikes can be reduced by $50 \%$ with even modest participation of users. Right: Sensitivity analysis for $\mathrm{p}_{1}\left(\right.$ with $\mathrm{p}_{2}=0$ )

\section{Discussion}

Our results suggest that user-based redistribution has a huge potential in free-floating BSS. Only considering drop-off incentives, we have shown that even with moderate participation of users, the number of necessary bikes to reach a specified service level can be substantially reduced. Since the total costs of BSS, to a large extent, are proportional to the number of deployed bikes, this directly translates in huge savings. Of course, incentives also incur costs. However, in the smide field test it turned out that the savings were a factor 8 higher than the incentive costs.

In this paper, we only showed results for constant and homogeneous demand and dropping patterns. However, the same approach was applied to time-varying and nonhomogeneous patterns with very similar results.

\section{References}

1. Guía metodológica para la implantación de sistemas de bicicletas públicas en España. Madrid: Instituto para la Diversificación y Ahorro de la Energía (IDAE) (2007).

2. Reiss, S., Bogenberger, K. Optimal Bike Fleet Management by Smart Relocation Methods. 2016 IEEE 19th Int. Conf. on Intelligent Transportation Systems (2016).

3. Todd, M., Xue, L., Barth, M.J.: User-based vehicle relocation techniques for multiplestation shared-use vehicle systems, TRB (2004).

4. Singla, A., Santoni, M., Bartók, G., Mukerji, P., Meenen, M., \& Krause, A. Incentivizing Users for Balancing Bike Sharing Systems. In AAAI., pp 723-729 (2015).

5. Pfrommer, J., Warrington, J., Schildbach, G., \& Morari, M.: Dynamic vehicle redistribution and online price incentives in shared mobility systems. IEEE Transactions on Intelligent Transportation Systems 15 (4), 1567-1578 (2014).

6. Fricker, C., \& Gast, N.: Incentives and redistribution in homogeneous bike-sharing systems with stations of finite capacity. EURO J. on Transp. and Logistics, 1-31. (2014)

7. Jouini O., Dallery Y.: Moments of first passage times in general birth-death processes. Math. Methods Oper. Res. (2008) 68(1):49-76 